\title{
DESIGN OF INTELLIGENT TRAFFIC LIGHT SYSTEM USING VISIBLE LIGHT COMMUNICATION
}

\author{
Preeti K S \\ M. Tech DCE, Department of ECE \\ BMSCE, Bangalore, Karnataka, India
}

\author{
Arathi R Shankar \\ Associate Professor \& Head, \\ M. Tech DCE, Department of ECE \\ BMSCE, Bangalore, Karnataka, India
}

\begin{abstract}
The concept of Visible Light Communication (VLC) in transport sector shapes introduction of Intelligent Transport System (ITS) using VLC. The Intelligent Traffic System (ITS) is broadly spread across the world to benefit the human kind for their safety in real-time applications. VLC is a substitute for RF in terms of bandwidth usage for various possible applications. Here in this work the design for Vehicle-to-Vehicle (V2V) and Infrastructure-to-Vehicle (I2V) Communication utilizing VLC is carried out. Various input \& output parameters which are enabled in this design shall be used to make the system more flexible for upcoming generation of vehicles. With use of design, output metrics such as SNR, transmitted power \& data, received power \& data are analysed. The sliding parameters incorporate Distance between transmitter and collector, Irradiance angle, spacing between detectors, $X$ of the receiver and $Y$ of the receiver. Likewise, detector position can swing from - 2 to $+2 m$ towards $X$ and $Y$ axis is designed \& accordingly change in the received power is observed. Also, Infrastructure-to-Vehicle (I2V) \& Vehicle-to-Vehicle (V2V) communication considering real time possible scenarios are investigated.
\end{abstract}

Keywords-I2V, V2V \& VLC

\section{INTRODUCTION}

Visible Light Communication (VLC) for the use of the Intelligent Transportation System (ITS) is a good up-andcomer invention. The Intelligent Transportation System is an invention that entails constructing the traffic on board and, along these lines, offering a healthy excursion to both street and automobile customers. As most traffic signal challenges, ITS implements correspondence and data advances to provide a solution to this blockage. ITS is a coordinated use of technologies that incorporates sophisticated techniques using computers, PCs, and sensors. It is the voice of cars, promotes eco-friendliness, and facilitates true traffic management and measurement. The significant uses of ITS are configured street specifications, disaster driver warning system, impact evasion gadget, complex and variable speed limit traffic signal structure. The quantity of automobiles designed per year using the transportation base. Subsequently, it is mandatory to continue to enhance the well-being and competence of the transport structure.

The set-up of remote innovation for vehicle interchanges for ITS is currently based on Dedicated Short Range Communication (DSRC), which is an innovation of $5.9 \mathrm{GHz}$ radio frequency (RF) [1-3] for vehicle conditions that enables interchanges between in-region vehicles and street-side structures. The invention of the DSRC helps a few implementations in vehicle conditions, such as a note of convergence effect and a notice of crisis slowing down [3]. Nevertheless, on dense roads where the quantity of vehicles is high [1-4], correspondence in vehicle conditions using RF invention routinely experiences poor bundle selection quality. In addition, for vehicular communications, the use of RF technology, which is typically omnidirectional, requires the challenge of visually identifying the location of the transmitters [1]. Additional VLC systems consisting of LEDs as transmitters and camera receivers aimed at $\mathrm{V} 2 \mathrm{~V}$ applications and their features and capabilities are taken into account [5]. A novel CMOS image sensor called an OCI has been developed and mounted in the camera receiver to achieve the $10 \mathrm{Mbps}$ class data rate and real-time LED detection. In the development of the OCI, two items are very important, i.e. the CPx for high-speed signal reception and the 1-bit flag image output feature for fast and efficient LED detection. Designing Vehicle-to-Vehicle information transmission under environmental conditions with usage of modified fixed decision threshold (MFDT) where Red LED is considered as the transmitter and a photodiode is utilized to separate the sent information utilizing MFDT. The proposed model is accurate and reliable under stormy weather [6]. In addition to investigating the potential capabilities of power line communications and white LED indoor communications for wide band access, the author addresses in paper [7] the concepts of the VLC system using white LEDs.

The rest of the paper is organised as follows: Section II describes the system while results and discussion are 
presented in Section III. Conclusions and future work are given in Section IV.

\section{PROPOSED SYSTEM}

All traffic information includes three main exercises - the assortment of traffic information, the reduction in traffic information and the investigation into traffic. Fundamental traffic information includes the vehicle's volume, class, speed and weight. As of now, contact type sensors are generally used for the assortment of traffic information, such as inductive circle indicators, pneumatic cylinders, piezo-electric links or strips, and capacitance strips.

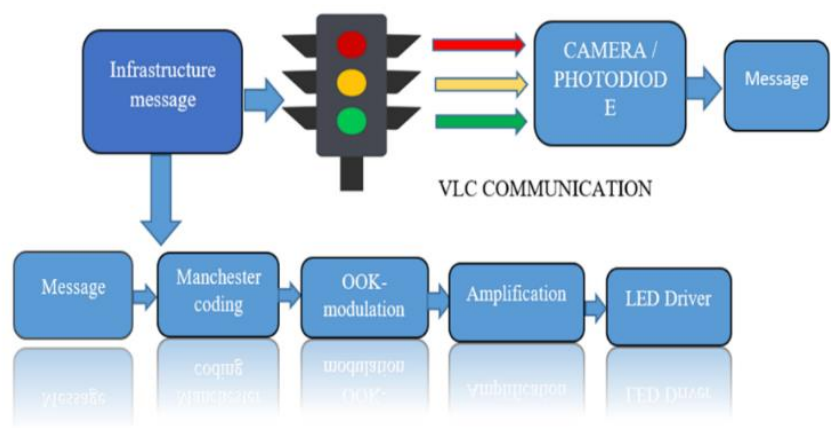

Fig. 1. I2V Communication

The I2V communication block diagram, shown in Fig 1, is mainly a message of infrastructure in which the information is sent to the traffic light signal, such as road conditions, traffic congestion, accidents and many additional information related to traffic safety. As it can be seen that the encoding of the message is done by Manchester code where the encoding of each data bit is low, high and low or equal to the same time in the Manchester code, also known as phase encoding, or PE.

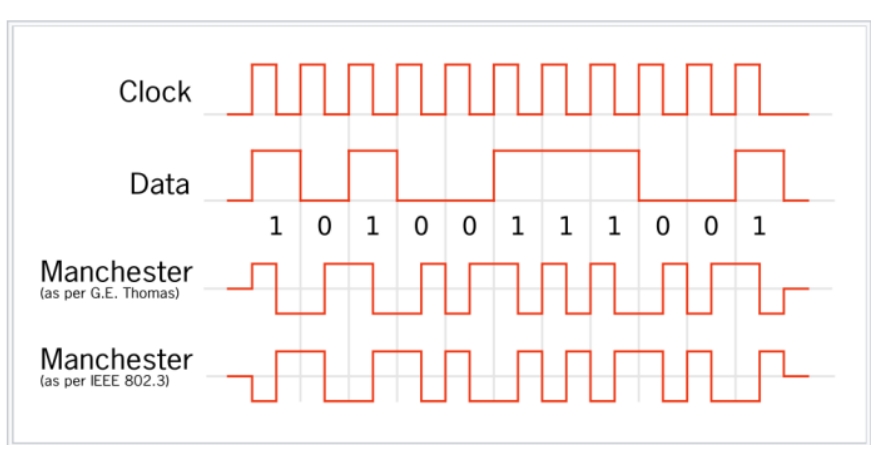

Fig. 2. An example of Manchester Coding

Manchester code has a transition at the middle of each bit period and may have a transition at the beginning of the period (depending on the information to be transmitted). The midbit transition direction shows the data. Transitions do not include details at time limits. They only work to position the signal in the right position to make a midbit transition. The modulative technique here is OOK (On-Off Key), in which OOK denotes the simplest mode of amplitude shift modulation (ASK) that represents the presence or absence of a carrier wave as digital data.

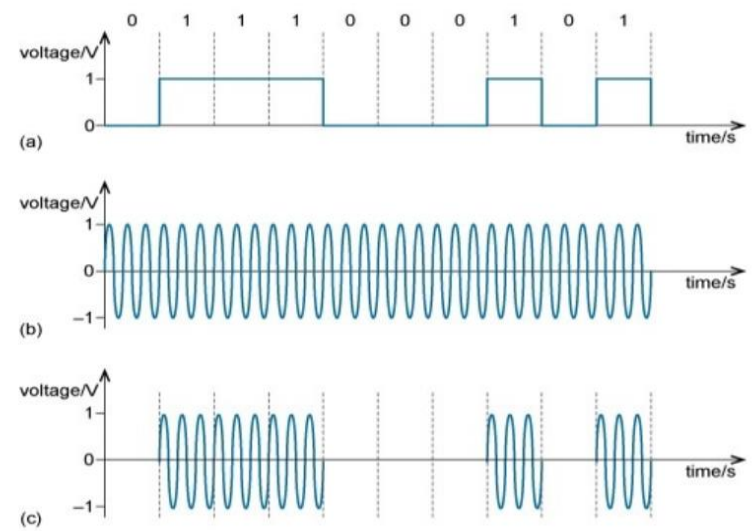

Fig. 3. An example of ON-OFF keying

As seen in Fig 3, the ON-OFF keyboard example shows that when the input signal combines with the carrier signal, the output is only achieved when the input signal is high. As a more energy-efficient light source, light-emitting diodes (LEDs) can soon replace fluorescent and incandescent lighting. LEDs can also be used to relay data at high speeds by modulating illumination, unlike fluorescent and incandescent lamps. The Lead is then used on the transmitter and the receiver uses the camera / photodetector to decipher the letter. Where the recipient segment is placed in the car to collect information relevant to the traffic. Manchester code decoding and OOK demodulation was done on the receiver.

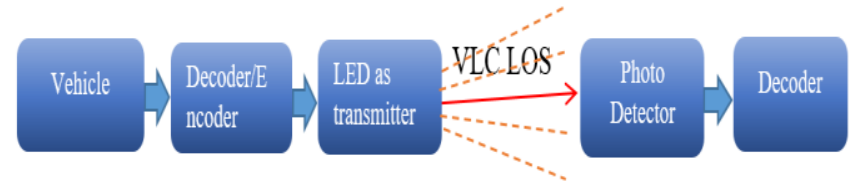

Fig. 4. V2V Communication

The trans receiver portion of $\mathrm{V} 2 \mathrm{~V}$ contact is shown in Fig 4. The vehicle's real-time traffic information or safety information is encoded using one of the coding methods where the Manchester coding is considered here. The message is transmitted to the vehicle's headlights, where the LED serves as the source of transmission into free space. Since the VLC deals with line of sight technologies, all the detectors within the transmitted beam coverage area will detect the data transmitted based on their sensitivity, but the detector that is directly in the transmitter's LOS can receive the exact data. This light beam is transformed into an electrical signal and decoded using the receiver's decoding module.

\section{$(2 \times 2)$ MIMO V2V-VLC System Model}




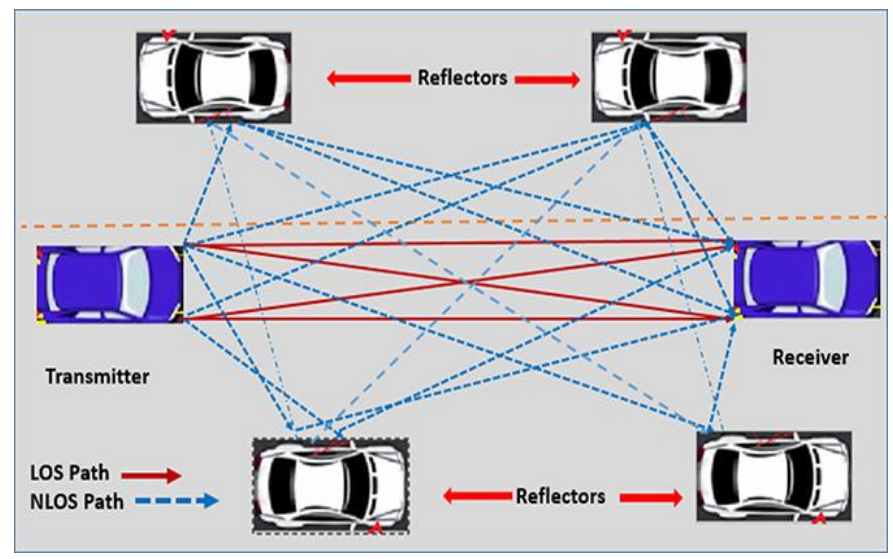

Fig. 5. $(2 \times 2)$ MIMO model for V2V-VLC showing the LOS and NLOS paths.

The V2V-VLC system shown in Figure 5 consists of two vehicles which, using headlights and taillights, communicate with each other. The first vehicle's headlights act as transmitters, and the second vehicle's taillights act as receivers. As each vehicle has two headlights and two taillights, it is possible to consider a 2 x 2 MIMO connection.

\section{System Model}

Figure 5 displays a block diagram of the V2V-VLC system. Using a Single-to-Parallel (S / P) converter, the transmitted serial data stream of the $2 \times 2$ MIMO model is translated into two parallel streams. Each stream of data is modulated by intensity and transmitted by LED headlights. PDs transform the light into electrical signals at the receiver taillights using direct tracking. It is necessary to write the obtained signal as:

$$
\mathrm{y}_{\mathrm{r}}(\mathrm{t})=\gamma \mathrm{x}(\mathrm{t}) \otimes \mathrm{h}(\mathrm{t})+\mathrm{n}(\mathrm{t})
$$

where $\mathrm{x}(\mathrm{t})$ is the transmitted signal, $\mathrm{y}_{\mathrm{r}}(\mathrm{t})$ is the received signal, $\mathrm{h}(\mathrm{t})$ is the CIR, $\gamma$ is the PD responsivity, $\otimes$ is the convolution sign, and $\mathrm{n}(\mathrm{t})$ is the noise.

\section{Channel Impulse Response and Transfer Function}

The total CIR of the V2V-VLC model, $h(t)$ is given by:

$$
\mathrm{h}(\mathrm{t})=\mathrm{h}(\mathrm{t})_{\mathrm{LOS}}+\mathrm{h}(\mathrm{t})_{\text {NLOS }}
$$

By performing Fast Fourier Transform (FFT) on above equation we get:

$$
\mathrm{H}(\dot{\omega})=\mathrm{H}(\dot{\omega})_{\mathrm{LOS}}+\mathrm{H}(\dot{\omega})_{\mathrm{NLOS}}
$$

\section{Impulse Response and Transfer Function of the LOS Component}

The $2 \times 2$ impulse response matrix HLOS between the transmitters and the receivers for the LOS path can be written as:

$$
\mathrm{H}_{\mathrm{rs}}(\mathrm{t})=\mathrm{G}_{\mathrm{rs}} \delta\left(\mathrm{t}-\tau_{\mathrm{rs}}\right) \quad \mathrm{r}=1,2 \quad \& \quad \mathrm{~s}=1,2
$$

where $G_{r s}$ denotes the gain factor accounting for the losses and gains between the source $s$ and the receiver $\mathrm{r}, \tau_{\mathrm{rs}}=\mathrm{R}_{\mathrm{rs}} / \mathrm{c}$, denotes the time delay, $\mathrm{R}_{\mathrm{rs}}$ denotes the distance between the source $\mathrm{s}$ and receiver $\mathrm{r}, \mathrm{c}$ is the speed of light, and $\delta()$ denotes the impulse function.

\section{RESULTS \& DiSCUSSION}

In proposed system the attempt to design V2V \& I2V Communication using VLC in simulation environment is done. With the use of UI based sliding parameters, output metrics such as SNR, received power, transmitted data from the LED'S and Received data from the detectors are analyzed. The results show an accuracy in received power and SNR up to $-60 \mathrm{db}$. In addition, enabling communication between one vehicle to another vehicle whose detector position can swing from -2 to $2 \mathrm{~m}$ across $\mathrm{X} \& \mathrm{Y}$ axis is implemented \& accordingly change in received power is observed.

\begin{tabular}{|l|l|}
\hline LED & $0.1 \mathrm{~W}$ \\
\hline Height of LED & $1.5 \mathrm{~m}$ (standard for simulation) \\
\hline Distance between TX \& RX & $2.5 \mathrm{~m}$ \\
\hline Modulation & OOK (on-off keying) \\
\hline Encoding & Manchester coding \\
\hline Photo detector refractive index & 1.46 \\
\hline Photo diode responsivity & 0.55 \\
\hline Data rate & $115200 \mathrm{~b} / \mathrm{s}$ \\
\hline Brightness factor & 4 \\
\hline LED viewing angle & 15 \\
\hline Photo diode FOV & 20 \\
\hline
\end{tabular}

Table. 1. List of simulation parameters with their range

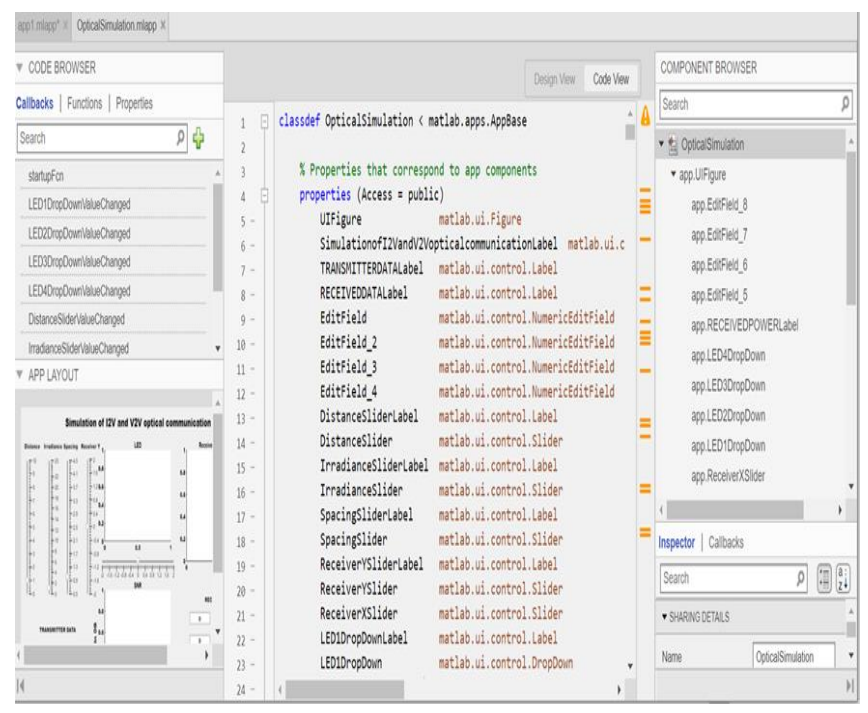

Fig. 6. Properties that correspond to app components Results for I2V Communication

Considering the following cases to demonstrate $\mathrm{I} 2 \mathrm{~V}$ communication and analysing output parameters.

Case 1: Distance and irradiance parameter of transmitter is fixed 


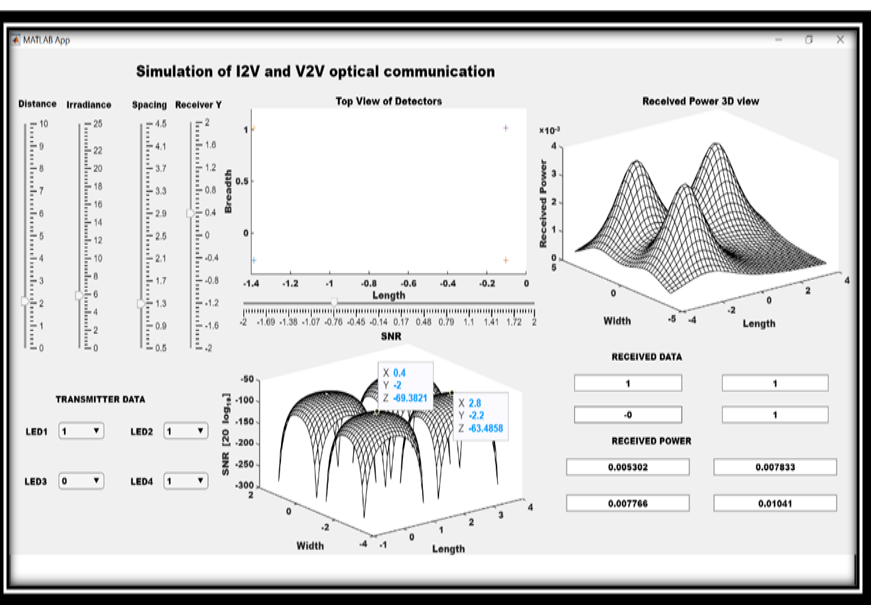

Fig. 7. Distance and irradiance parameter of transmitter is fixed

The transmitter parameters are set as seen in Fig 7 to arrive at I2V contact, such as the distance between transmitter and receiver and the transmitter irradiance angle is fixed and variation on the receiver side is carried out. Initially, as seen above, we analyse parameters by sliding them to a specific value where we can analyse encoded data, SNR \& Obtained power.

Case 2: Distance and irradiance are fixed $\&$ varying $X$ of receiver

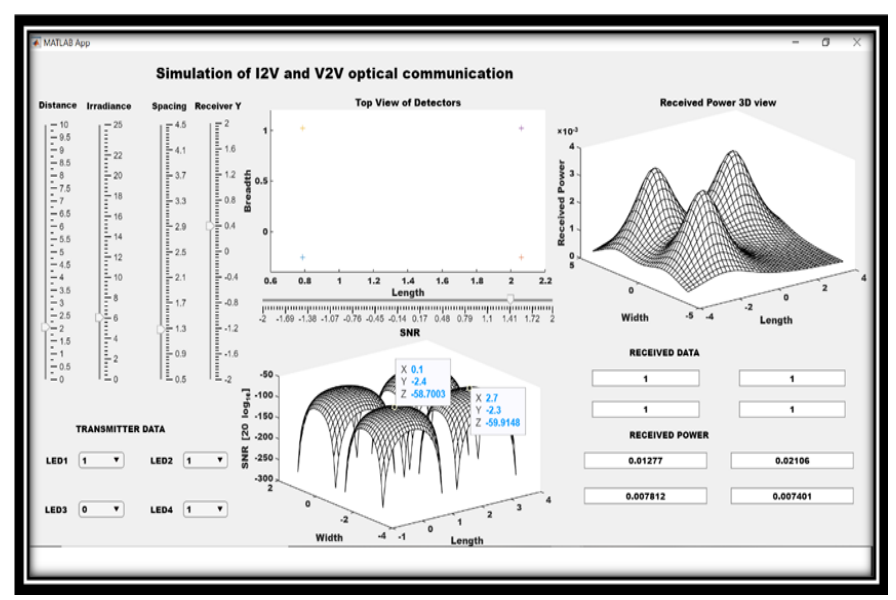

Fig. 8. Distance and irradiance are fixed \& varying $X$ of receiver

Considering case 2 w.r.t case 1 no parameter is varied except increasing $X$ of the receiver. The $X$ of the receiver basically signifies the position of the receiver at $\mathrm{X}$ axis i.e. distance. So by increasing the value of $X$ of receiver we can see that SNR of case 1 is around $-65 \mathrm{db}$ which is decreased $(-58 \mathrm{db})$ in case $2 \&$ also corruption of data is observed. There by the received power also changes as we vary $\mathrm{X} \& \mathrm{Y}$ of the receiver according to constructive and distractive interference's.

Case 3: Distance and Irradiance is fixed \& $\mathrm{Y}$ of receiver is varied

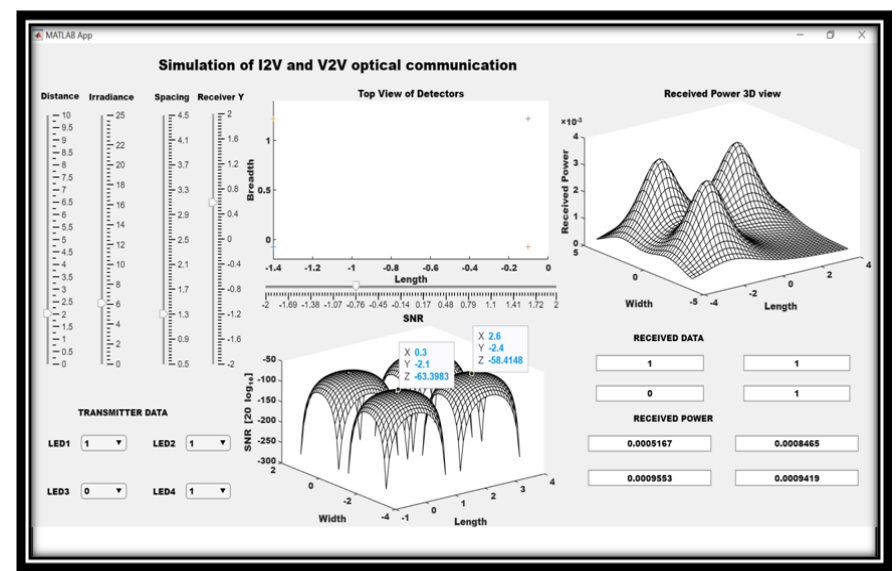

Fig. 9. Distance and Irradiance is fixed $\& Y$ of receiver is varied

Considering case 3 w.r.t case 1 where all the parameters are same except increasing the $\mathrm{Y}$ of the receiver. $\mathrm{Y}$ of receiver basically signifies the position of the detectors at $y$ position. This parameter broadens the application of the communication by enabling the communication between one vehicle to another vehicle whose detector position swings from $-2 \mathrm{~m}$ to $+2 \mathrm{~m}$ upside-down. Accordingly, output parameters are analysed if the detector position is properly fixed for any of the vehicle and if it is with LOS with the LED then we can expect no loss of information with high SNR (-63db). Also the received power changes when varying $\mathrm{Y} \& \mathrm{X}$ of receiver according constructive and destructive interference.

\section{Results for V2V Communication}

Case 1: For V2V communication initially all input parameters are varied.

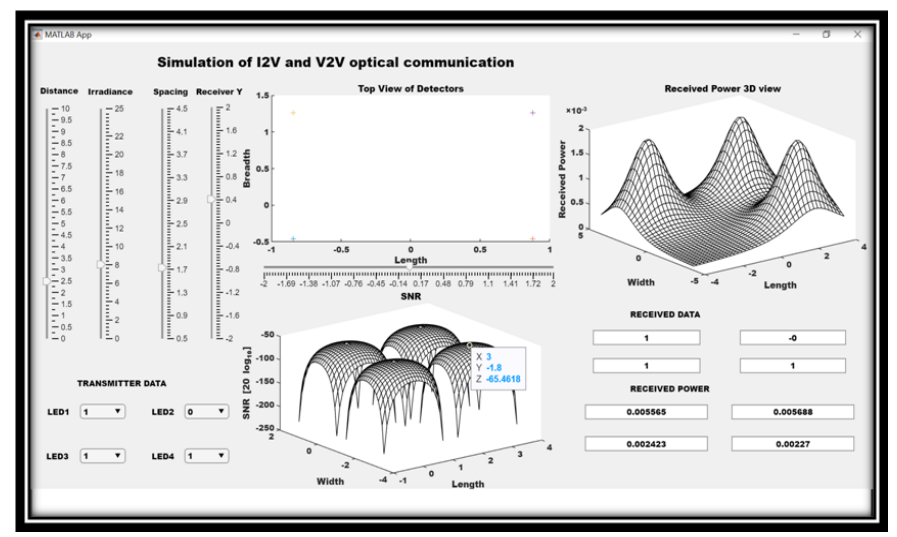

Fig. 10. For V2V communication initially all input parameters are varied

Considering case 1 for $\mathrm{V} 2 \mathrm{~V}$ communication where the initial setup by fixing the input parameters to particular value where we can analyse the output parameters is done. We can analyse that when distance between transmitter and receiver is less and keeping the irradiance also less we can get accurate data at receiver also increases SNR $(-65 \mathrm{db})$ of the system. The received power also varies according to $\mathrm{x}$ and $\mathrm{y}$ position of the receiver. 
Case 2: Distance at transmitter side and receiver side is varied

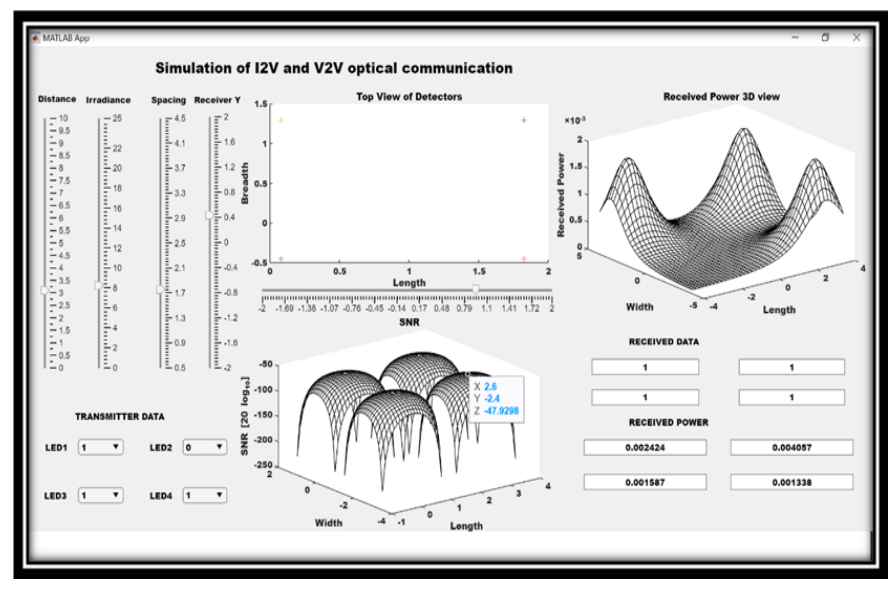

Fig. 11. Distance at transmitter side and receiver side is varied

Considering the case 2 w.r.t case 1 all parameters are kept same except distance and $\mathrm{X}$ of receiver is varied. By more increase in distance between transmitter and receiver the SNR is reduced to $-47 \mathrm{db}$ with corruption of the transmitted data at the receiver is seen. The received power is also decreased when compared to above case.

\section{Case 3: Irradiance angle is decreased}

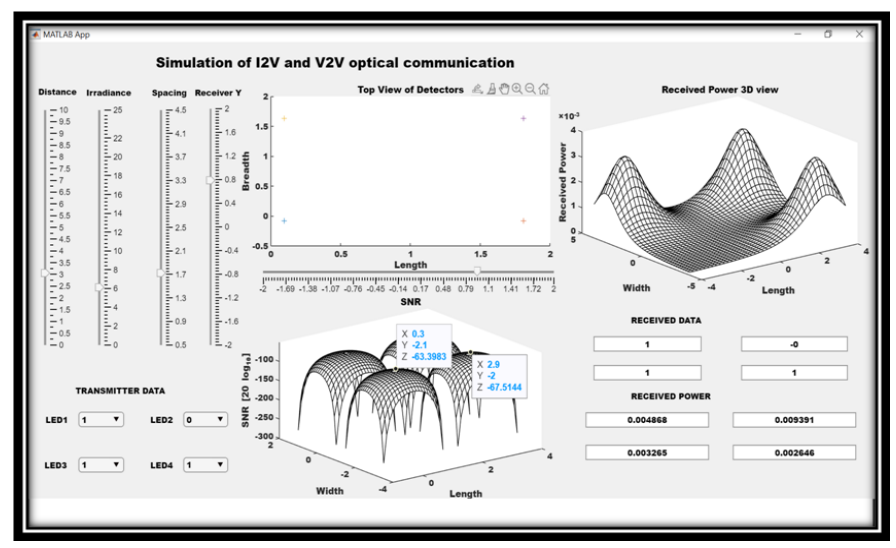

Fig. 12. Irradiance angle is decreased

Considering case 3 w.r.t case 2 all the parameters remains same except decreasing the irradiance angle. As the angle of irradiance between transmitter and absorbing component is less and is in LOS with detector there is high $\operatorname{SNR}(-67 \mathrm{db})$ obtained with no loss of data.

\section{Case 4: Irradiance angle is increased}

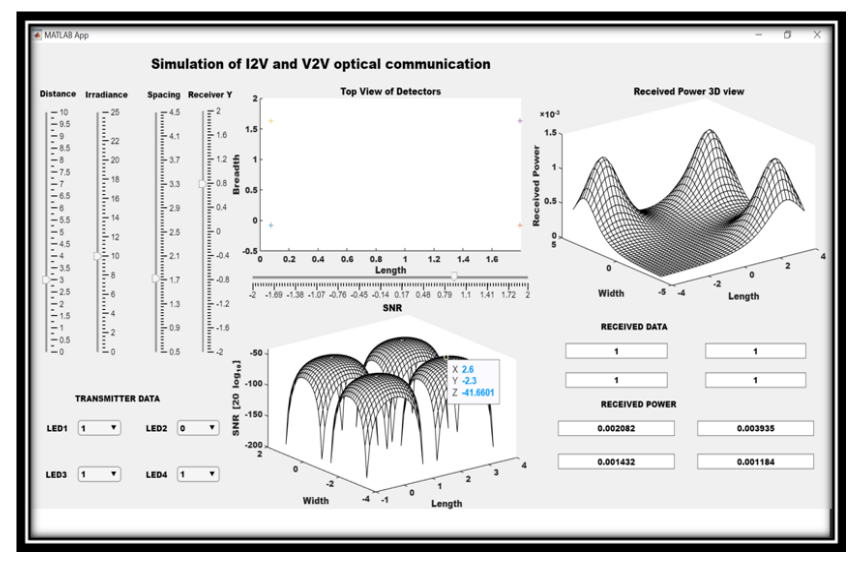

Fig. 13. Irradiance angle is increased

Considering case 4 w.r.t case 3 where all the parameters are same except irradiance angle is increased. Comparing case 5 and 6 one can analyse that by increasing the irradiance angle keeping same distance between transmitter and receiver the SNR(-41db) decreases. The received power also decreased when compared with the case 3 .

\section{CONCLUSION \& FUTURE WORK}

The usage VLC in transport sector leads to Intelligent Transport System (ITS). The ITS is widely distributed across the globe in order to eventually support humanity for their well-being applications. In this work the attempt to design V2V \& I2V Communication using VLC in simulation environment is done. Different information and yield boundaries which are empowered in this design will be utilized to make the framework more adaptable for forthcoming age of vehicles. Further various possible cases for $\mathrm{I} 2 \mathrm{~V}$ and $\mathrm{V} 2 \mathrm{~V}$ communication are considered where we can analyse targeted output parameters such as SNR, received power, transmitted information from the LED'S and received information from the detectors. The UI based sliding input parameters which are enabled in this design covers various possible cases which shows demonstration of real time scenario's for I2V \& V2V communication. Also enabling communication between one vehicle to any of the on road vehicle whose receiver position can swing from -2 to $+2 \mathrm{~m}$ towards $\mathrm{X}$ and $\mathrm{Y}$ axis is designed \& accordingly, change in the received power is observed.

The study which has been investigated here is about ITS of enabling $\mathrm{I} 2 \mathrm{~V} \& \mathrm{~V} 2 \mathrm{~V}$ commination using VLC. Job architecture is carried out by considering different input parameters and studying different output parameters. But there are certain limitations, which are listed below. These provide a scope for further investigation.

- The enhancement of the work done in this paper can be carried out to make the system suitable for real-time working scenario's. 
- This paper gives the first attempt in implementing Application for ITS by using basic modulation and encoding techniques. Future work can be carried out by designing the system using various other modulation \& encoding techniques which can be well used in future transport system to ensure safety of mankind.

- Various input \& output parameters which are enabled in this Application shall be used to make the system more flexible for upcoming generation of vehicles. One shall also continue this work by considering climatic conditions, metropolitan areas \& communication when humps, cross overs are considered.

\section{REFERENCE}

[1] W. Shen and H. Tsai, (2017) "Testing vehicle-to-vehicle visible light communications in real-world driving scenarios," in 2017 IEEE Vehicular Networking Conference (VNC), pp. (187-194).

[2] N. Vivek, S. V. Srikanth, P. Saurabh, T. P. Vamsi, and K. Raju, (2014) "On field performance analysis of IEEE 802.11p and WAVE protocol stack for V2V \&amp; V2I communication," in International Conference on Information Communication and Embedded Systems (ICICES2014), pp. 16

[3] S. Gao, A. Lim, and D. Bevly, (2016) "An empirical study of DSRC V2V performance in truck platooning scenarios," Digital Communications and Networks, vol. 2, pp. 233-244.

[4] Z. Xu, X. Li, X. Zhao, M. H Zhang, and Z. Wang, (2017) "DSRC versus 4GLTE for Connected Vehicle Applications: A Study on Field Experiments of Vehicular Communication Performance" vol. 435, 2017.

[5] MEHBOOB RAZA HAIDER, MANOJ M. DONGRE, (2015) "VEHICLE TO VEHICLE COMMUNICATION USING "VISIBLE LIGHT COMMUNICATION TECHNOLOGY" Proceedings of 24th IRF International Conference, pp. 64-66

[6] Y. H. Kim , W. A. Cahyadi and Y. H. Chung, (2015) "Experimental demonstration of LED based vehicle to vehicle communication under atmospheric turbulence, "In proceedings of International Conference on Information and Communication Technology Convergence (ICTC), Jeju, pp. 1143- 1145

[7] P. Amirshahi and M. Kavehrad, (2006) "Broadband access over medium and low voltage powerlines and use of white light emitting diodes for indoor communications," In proceedings of IEEE Consumer Communications and Networking Conference (CCNC), University park, PA, pp. 897- 901
[8] P. Luo, Z. Ghassemlooy, H. L. Minh, E. Bentley, A. Burton, and X. Tang, (2015) "Performance analysis of a carto-car visible light communication system," Applied Optics, vol. 54, pp. 1696-1706

[9] W. H. Organization, "World Health Organization Global Status Report on Road Safety 2018," World Health Organization 2018.

[10] Elizabeth Eso, Zabih Ghassemlooy, Stanislav Zvanovec, Asghar Gholami (2019) "Experimental Demonstration of Vehicle to Road Side Infrastructure Visible Light Communications" The 2nd West Asian Colloquium on Optical Wireless Communications (WACOWC2019) 27-28 April, Shahid Beheshti University, IEEE, pp. 85-89

[11] Hasan Faarahneh , (2004) "INVESTIGATION OF VEHICLE TO VEHICLE COMMUNICATION SYSTEM USING VISIBLE LIGHT TECHNOLOGY"

[12] Y. H. Kim ,W. A. Cahyadi and Y. H. Chung, (2015) "Experimental demonstration of LED based vehicle to vehicle communication under atmospheric turbulence, "In proceedings of International Conference on Information and Communication Technology Convergence (ICTC), Jeju, pp. 1143- 1145 